\title{
BMJ Detection and follow-up of Open cardiovascular disease and risk factors in the Southern Cone of Latin America: the CESCAS I study
}

Adolfo Luis Rubinstein, ${ }^{1}$ Vilma Edith Irazola, ${ }^{1}$ Rosana Poggio, ${ }^{1}$ Lydia Bazzano, ${ }^{2}$
Matías Calandrelli, ${ }^{3}$ Fernando Tomas Lanas Zanetti, ${ }^{4}$ Jose Anibal Manfredi, ${ }^{5}$
Héctor Olivera, ${ }^{6}$ Pamela Seron, ${ }^{5}$ Jacqueline Ponzo, ${ }^{7}$ Jiang $\mathrm{He}^{2}$

To cite: Rubinstein $\mathrm{AL}$, Irazola VE, Poggio $\mathrm{R}$, et al. Detection and follow-up of cardiovascular disease and risk factors in the Southern Cone of Latin America: the CESCAS I study. BMJ Open 2011;1:e000126.

doi:10.1136/bmjopen-2011 000126

- Prepublication history for this paper is available online. To view these files please visit the journal online (http:// bmjopen.bmj.com).

Received 24 March 2011 Accepted 4 April 2011

This final article is available for use under the terms of the Creative Commons Attribution Non-Commercial 2.0 Licence; see http://bmjopen.bmj.com

For numbered affiliations see end of article.

Correspondence to Adolfo Luis Rubinstein; arubinstein@iecs.org.ar

\section{ABSTRACT}

Introduction: Cardiovascular diseases (CVD) are increasing throughout the world and cause 16.7 million deaths each year, $80 \%$ of which occur in low and middle income countries. In Argentina, Chile and Uruguay, the available data on cardiovascular risk factors come predominantly from cross-sectional studies that are principally based on self-report or studies conducted with small convenience samples. The CESCAS I study will generate reliable estimates of the prevalence and distribution of and secular trends in CVD and its risk factors in this region.

Methods and analysis: CESCAS I is an observational prospective cohort study with a multistage probabilistic sample of 8000 participants aged 35-74 years from four mid-sized cities representing the Southern Cone of Latin America: Bariloche and Marcos Paz in Argentina, Temuco in Chile and Pando-Barros Blancos in Uruguay. In the first phase, baseline data regarding exposure to risk factors and prevalence of CVD will be collected in two stages: (1) in homes and (2) in health centres. Information will be gathered on medical history, risk factors, lifestyles and health utilisation through specific questionnaires, physical measurements, an ECG and an overnight, fasting blood sample to measure levels of serum lipids, glucose and creatinine. In the second phase, annual follow-up data will be obtained on the incidence rate of CVD events and the association between exposure and events.

Ethics and dissemination: The protocol has obtained formal ethics approval from institutional review boards in Argentina, Chile, Uruguay and the USA. The lack of follow-up studies has prevented Argentina, Chile and Uruguay from implementing risk factor stratification and management strategies at a population level. However, the CESCAS I study data will help the development of public health strategies based on primary care intervention, thus helping to improve cardiovascular health in this region.

\section{ARTICLE SUMMARY}

Article focus

- To estimate the prevalence and distribution of and secular trends in major cardiovascular disease (CVD) events and risk factors in four cities in Argentina, Chile and Uruguay.

Key messages

- Lack of follow-up studies prevents Argentina Chile and Uruguay from assessing local risk estimates, obtaining reliable data on CVD burden and implementing risk factor stratification and management strategies at a population level.

- There is a strong need in the region to build the capacity and infrastructure to undertake a population-based cohort study to address knowledge gaps and to inform policymakers of the impact of CVD in the Southern Cone of Latin America.

Strengths and limitations of this study

- CESCAS I will be the first longitudinal study to estimate the CVD trend and risk factors in Argentina, Chile and Uruguay.

\section{INTRODUCTION}

Cardiovascular diseases (CVD) are increasing throughout the developing world and cause almost 16.7 million deaths each year, $80 \%$ of which occur in low and middle-income countries. ${ }^{1}$ Indeed, $34 \%$ of annual deaths are due to CVD. ${ }^{2}$ It has been projected that 41 million people world-wide will die in 2015 of chronic CVD unless effective concerted action is taken now. ${ }^{3}$ In low and middleincome countries, almost half of these deaths will occur in people below 70 years of age compared with only $27 \%$ in high-income countries. ${ }^{4}$ Furthermore, although in recent 
decades age-adjusted rates for cardiovascular mortality have decreased in developed countries, rates have increased in low and middle-income countries. ${ }^{15}$

In Latin America, it is estimated that between 1990 and 2020, deaths from CVD, including coronary heart disease (CHD), will increase by approximately $145 \%$ in both men and women compared with an increase of $28 \%$ in women and an increase of $50 \%$ in men in developed countries during the same period. ${ }^{6}$

Moreover, at least $75 \%$ of CVD can be explained by risk factors such as an unhealthy diet, low physical activity and tobacco use. ${ }^{7}$ In the World Health Report 2002, 26 risk factors were evaluated and ranked by their importance. Major risk factors identified for most Latin American countries were hypertension, increased body mass index (BMI), and alcohol and tobacco use. ${ }^{8}$ The Latin American INTERHEART study showed that most cardiovascular risk in the Southern Cone could be explained by tobacco use, abnormal lipids, abdominal obesity and high blood pressure. ${ }^{9}$ In Argentina, recent estimates showed that in 2005 there were more than 600000 disability adjusted life years (DALYs) and almost 400000 years of potential life lost due to CHD and stroke, where modifiable risk factors explained $75 \%$ of fatal and non-fatal acute CHD and stroke events, $82 \%$ of acute CHD events and $62 \%$ of strokes. Similarly, modifiable risk factors explained $76 \%$ of costs due to acute events and $71 \%$ of DALYs lost. ${ }^{10}$ In Argentina, Chile and Uruguay, the available data on CVD risk factors come mainly from cross-sectional studies that are principally based on self-report or studies conducted with small convenience samples, which do not give reliable estimates. ${ }^{11-13}$ None of the studies mentioned above included a prospective follow-up study. While the ongoing ELSA study, a recently established cohort study in Brazil, will provide data on cardiovascular events, its population is composed of employee volunteers from six Brazilian universities and thus will not be able to offer populationbased estimates of the impact of CVD risk factors on the incidence of CVD. ${ }^{14}$

The CESCAS I study will generate reliable estimates of the prevalence and distribution of and secular trends in CVD and its risk factors in this region. These data will contribute to the development of public health strategies, thus helping to improve cardiovascular health in Latin America.

\section{METHODS}

\section{Study design}

CESCAS I is an observational, prospective cohort study initiated in November 2010 and is composed of two phases. In the first phase, baseline data will be collected regarding exposure to risk factors and prevalence of CVD. In the second phase, annual follow-up data will be obtained on the CVD incidence rate and the association between exposure and the event.

\section{Study population}

This study entails a probabilistic sample of 8000 noninstitutionalised mainly urban men and women between the ages of 35 and 74 years representing the general adult population in Argentina, Chile and Uruguay. Four mid-sized cities have been selected: two in Argentina (Bariloche and Marcos Paz), one in Chile (Temuco) and one in Uruguay (Pando-Barros Blancos).

\section{Sampling method}

As the prevalence of CVD risk factors was considered likely to vary by age, gender and geographical area, the sampling method was stratified accordingly, following a complex sampling design that consisted of four stratified stages to obtain a representative sample from each of the four locations (figure 1). The first stage consisted of randomly sampling census radii from each location, stratified by socio-economic level. In the second stage a number of blocks proportional to the radius size were randomly selected. The third stage sampled households from each block using systematic sampling. If the selected house did not include a permanent residence (eg, offices/weekend residences, abandoned or demolished dwellings, those under construction, or addresses which were not identified), it was replaced with another house. In the selected households all members between 35 and 74 years of age were recorded to create the final sampling frame. In the fourth stage, one recorded member per household was randomly selected. The final sampling frame was composed of one subject per household, stratified by gender (50\% women and $50 \%$ men) and age category (35-44, 45-54, 55-64 and 65-74 years old) constituting a total of 2000 subjects per site, all of them meeting the following criteria: a permanent resident at the location for at least 6 months per year, willing to sign a consent form to participate, not intending to relocate within the next 2 years, and able to respond autonomously to the questionnaire (without cognitive impairment or language problems). Replacement of selected participants because of refusal to participate or inability to be located was not allowed. ${ }^{15} 16$

\section{Recruitment plan}

Participants are invited to participate through a letter from the site institution. The interviewer makes the first contact with the household and arranges an appointment for a home visit to collect questionnaire data and schedule a follow-up clinic visit. A clinic visit is scheduled

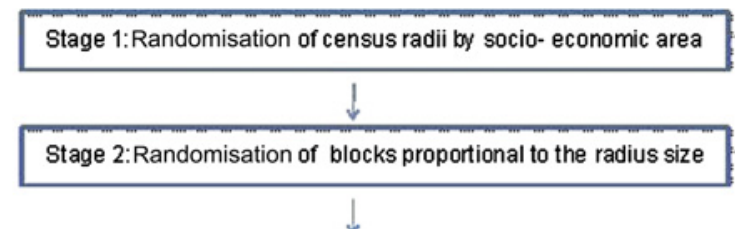

Stage 3: Systematic selection of households by census radius/housholds listing

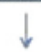

Stage 4:Randomisation of subjects Total sample: 8.000

Figure 1 Sampling procedure in the CESCAS I study. 
to obtain physical measurements, an ECG and overnight fasting blood samples. To minimise non-participation and the potential for bias, the following measures will be taken to facilitate and encourage participation:

- Travel assistance or home assessment for those unable to travel to the examination centres

- Variety of appointment times to suit all members of the community

- Feedback of blood test and examination results to participants.

\section{Data collection}

Baseline data collection is conducted in two stages: at home and in the health centre. In the home, an interviewer administers specific questionnaires to collect the required information. Once the survey is complete, the interviewer proceeds to arrange a visit to a health centre where the physical measurements, ECG and blood sample will be obtained.

\section{Questionnaires}

A trained interviewer will gather information regarding participant characteristics including demographic, socioeconomic and healthcare utilisation data, personal and family history of CVD, and risk factors such as high blood pressure, dyslipidaemia and diabetes, as well as current pharmacological and non-pharmacological treatment. Data will also be collected regarding intermittent claudication, cancer, respiratory disease, alcohol consumption and weight history using cross-culturally adapted questionnaires from the Hispanic Community Health Study/Study of Latinos (HCHS/SOL). ${ }^{17}$ Physical activity will be assessed through the HCHS/SOL study questionnaire adapted from the International Physical Activity Questionnaire. ${ }^{18}$ Information about current and former cigarette smoking, including age at which smoking was initiated, years of smoking, number of cigarettes smoked per day, cessation attempts and treatments, will be assessed using the Global Adult Tobacco Survey. ${ }^{19}$ Use of other forms of tobacco, exposure to passive cigarette smoking and indoor pollution will also be assessed. Nutritional information will be collected using a semi-quantitative food frequency questionnaire adapted from the National Cancer Institute Diet History Questionnaire, which has been validated by our research team for use in Argentina, Chile and Uruguay ( $\mathrm{N}$ Elorriaga, personal communication, 2011). Depression and anxiety will be assessed by the nine-item Patient Health Questionnaire (PHQ-9), ${ }^{20}$ which has been validated in Argentina. ${ }^{21}$ Stressful events and spirituality will be assessed through a crossculturally adapted version of the HCHS/SOL study questionnaire. $^{17}$

Locally validated versions of the SF-12 and EQ-5D will be used to measure health-related quality of life and social utilities and preferences, respectively. ${ }^{22}{ }^{23}$ All questionnaires used in the study and their sources are listed in table 1.
Blood pressure and anthropometric measurements

Trained and certified observers will measure blood pressure during the health centre visit following the recommendations of the American Heart Association. ${ }^{24}$ According to the study protocol, before blood pressure is measured, the participant should remain seated and at rest for $5 \mathrm{~min}$. Tea, mate or coffee consumption, as well as smoking or exercising, in the $30 \mathrm{~min}$ before testing is not permitted. A standardised mercury or aneroid sphygmomanometer with an adequate cuff size will be used. The cuff will be placed on the right arm of the participant, inflated to $10 \mathrm{~mm} \mathrm{Hg}$, and then inflated again until it reaches a pressure $30 \mathrm{~mm} \mathrm{Hg}$ above the level at which the radial pulse can no longer be palpated. Three measurements will be obtained, with $30 \mathrm{~s}$ intervals between them. Korotkoff sounds will be recorded, and used to identify systolic and diastolic blood pressure.

Weight will be measured with the subject wearing undergarments but not shoes. Weight will be recorded in kilograms to one decimal place using standing scales supported on a steady surface. Height will be measured without shoes, in centimetres to one decimal place, on the Frankfort plane positioned at a $90^{\circ}$ angle against a metallic metric tape measure mounted on a wall. Abdominal circumference will be measured in centimetres to one decimal place, on a horizontal plane $1 \mathrm{~cm}$ above the belly button which generally coincides with the narrowest waist circumference.

\section{Laboratory measurements}

Overnight fasting blood samples will be drawn by venipuncture to measure levels of serum lipids, glucose and creatinine. Samples will be processed and temporarily stored at the extraction site to be sent later for analysis and storage (in ultra freezers at $-80^{\circ} \mathrm{C}$ ) in the central laboratory at the Hospital Italiano of Buenos Aires. LDL cholesterol levels will be calculated using the Friedewald equation for participants with triglyceride levels $<400 \mathrm{mg} / \mathrm{dl}$. According to this equation, total LDL cholesterol is equal to: total cholesterol-HDL cholesterol-triglycerides/5.

\section{Electrocardiogram}

The study will employ a 12-lead electrocardiogram standardised at $25 \mathrm{~mm} / \mathrm{s}$ and at $1 \mathrm{mV}$ of amplitude.

\section{Study outcomes}

Hypertension is defined as a mean systolic blood pressure $\geq 140 \mathrm{~mm} \mathrm{Hg}$ and/or diastolic blood pressure $\geq 90 \mathrm{~mm} \mathrm{Hg}$, and/or self-report of current use of antihypertensive medications. ${ }^{25}$ Obesity is defined as BMI $\geq 30 \mathrm{~kg} / \mathrm{m}^{2}$ and overweight as BMI $\geq 25 \mathrm{~kg} / \mathrm{m}^{2}$. ${ }^{26-29}$ Dyslipidaemia is defined as total cholesterol $\geq 200 \mathrm{mg} / \mathrm{dl}$ or $11.1 \mathrm{mmol} / \mathrm{l}$, LDL cholesterol $\geq 130 \mathrm{mg} / \mathrm{dl}$ or $7.2 \mathrm{mmol} / \mathrm{l}$, or HDL cholesterol $<40 \mathrm{mg} / \mathrm{dl}$ or $2.2 \mathrm{mmol} / \mathrm{l}^{30}$ Diabetes mellitus is defined as fasting glucose $\geq 126 \mathrm{mg} / \mathrm{dl}$ or $7 \mathrm{mmol} / \mathrm{l}$. Glucose intolerance is defined as a fasting glucose level of $110-125 \mathrm{mg} / \mathrm{dl}$ or $6.1-6.9 \mathrm{mmol} / \mathrm{l}^{31}$ Current smoking is defined as 
Table 1 Data collection in the CESCAS I study

\begin{tabular}{|c|c|c|}
\hline Type of data & Components & Instrument \\
\hline General information & $\begin{array}{l}\text { Socio-demographic and economic data, and type of health services } \\
\text { utilisation }\end{array}$ & $\mathrm{HCHS} / \mathrm{SOL}^{*}$ \\
\hline Claudication & Location, functional class & $\mathrm{HCHS} / \mathrm{SOL}^{*}$ \\
\hline History & $\begin{array}{l}\text { Cardiovascular, respiratory, hypertension, dyslipidaemia, diabetes, } \\
\text { pharmacological and non-pharmacological treatment and cancer }\end{array}$ & $\mathrm{HCHS} / \mathrm{SOL}^{*}$ \\
\hline Alcohol & $\begin{array}{l}\text { Level of consumption (daily quantity, frequency, type of alcoholic } \\
\text { beverage) }\end{array}$ & $\mathrm{HCHS} / \mathrm{SOL}^{*}$ \\
\hline Physical activity & Type of activity, frequency and intensity, in free time and during work & IPAQ \\
\hline Spirituality & Importance, religious practice & $\mathrm{HCHS} / \mathrm{SOL}^{*}$ \\
\hline Nutrition & Types of foods, quantity and frequency & FFQ \\
\hline Smoking & $\begin{array}{l}\text { Current, former and passive smoker; other types of tobacco use } \\
\text { (pipe, cigar) }\end{array}$ & GATS \\
\hline Mental health & Depression, traumatic events, anxiety & PHQ-9/HCHS/SOL* \\
\hline Global health & & SF-12 \\
\hline Quality of life & & EQ-5D \\
\hline Physical examination & Blood pressure, weight, height and waist circumference & \\
\hline Laboratory & Total cholesterol, HDL cholesterol, triglycerides, glucose and creatinine & \\
\hline ECG & $25 \mathrm{~mm} / \mathrm{s}$ and at $1 \mathrm{mV}$ of amplitude & \\
\hline
\end{tabular}

smoking at least one cigarette per day at the time of the survey. Former smoking is defined as a person who has been a smoker but does not smoke at the time of the survey. Passive smoking is defined as involuntarily breathing air that is contaminated by tobacco smoke. ${ }^{27} 32$

During the follow-up a person will be categorised as having CVD if it is self-reported or their self-assigned proxy has reported any of the following conditions diagnosed by a physician: CVD death, acute myocardial infarction, angina, heart failure, cerebrovascular events, peripheral vascular disease or coronary or peripheral revascularisation. The study outcome committee will review the collected source documents related to the reported event and adjudicate on all CVD outcomes independently.

\section{Training and quality assurance}

Field work quality assurance

Data collection will be conducted according to the standardised operations manual. Between $5 \%$ and $10 \%$ of participants will have repeated measurements of arterial pressure, height, weight and waist circumference to adjust for the effect of measurement error on selected variables. All the equipment used will be certified to international standardisation norms. Periodic monitoring of data collection procedures will be performed by the coordinating centre.

\section{Laboratory quality control}

All laboratory measurements (total cholesterol, HDL, LDL, triglycerides, glucose and plasma creatinine) will be carried out by the central laboratory. Each laboratory technician will have to complete a training program.

\section{Processing of data}

The database was designed using the OpenClinica system. $^{33}$ This is a computerised system of related databases with web interface. The data will be entered from each site, via the web, into electronic forms. Double data entry with independent operators will be performed to eliminate data entry errors. The data will be stored on a central server. Validation rules will be generated in accordance with the nature of the variables. Automatic queries will be generated in response to outof-range entries to be investigated by study personnel at each site.

\section{Statistical investigation \\ Sample size}

The calculated sample size is 8000 participants (2000 per site) which is consistent with the recommended requirements for precision for complex surveys. This sample will be sufficient to provide precise estimates of the prevalence of major CVD risk factors by gender and site, in four age-defined categories (35-44, 45-54, 55-64 and 65-74 years old) as well as their association with the development of CVD. ${ }^{34-36}$ The proposed sample size is sufficient to comply with the precision requirements of a complex sample that assumes that the design effect is 1.5 and the prevalence of the risk factors of interest is $5 \%$ or greater (table 2).

For analysis, the capacity to detect risk factors was calculated using a statistically significant $\alpha$ level of 0.05 and a statistical power of $85 \%$, which will permit detection of moderate and large relative risks.

\section{Statistical analysis}

The general characteristics of the population will be described. For continuous variables, mean and median, range, standard deviation and/or quartile range will be calculated according to the distribution of each variable. In the case of categorical variables, absolute and relative frequencies will be calculated. 
Table 2 Sample size required for different proportions and design effects

\begin{tabular}{lrrrrr}
\hline & \multicolumn{5}{c}{ Design effect } \\
\cline { 2 - 6 } Proportion & $\mathbf{1 . 0}$ & $\mathbf{1 . 5}$ & $\mathbf{2 . 0}$ & $\mathbf{2 . 5}$ & $\mathbf{3 . 0}$ \\
\hline $0.26-0.50$ & 30 & 45 & 60 & 75 & 90 \\
0.25 & 32 & 48 & 64 & 80 & 96 \\
0.20 & 40 & 60 & 80 & 100 & 120 \\
0.15 & 53 & 80 & 107 & 133 & 160 \\
0.10 & 80 & 120 & 160 & 200 & 240 \\
0.05 & 160 & 240 & 320 & 400 & 480 \\
\hline
\end{tabular}

In order to determine the prevalence and incidence of risk factors, CVD events and the association between risk factors and CVD events, the design effect of the first stage unit of sampling will be considered. Weighting will be based on the relationship between the number of individuals finally included in the study and the population size and composition of each site according to the most recent census data. Likewise, the analysis will be carried out by socioeconomic strata, according to gender and four age categories (35-44, 45-54, 55-64 and 65-74 years old).

To assess associations between risk factors and CVD events, linear regression and simple and multiple logistic regressions will be used according to the nature of the response variables. Continuous variables that are not normally distributed will be evaluated by the application of transformations and categorisations wherever applicable. $^{37}$

The secular trends in risk factors over time will be evaluated with statistical analysis methods that take account of the correlation between repeated measures. To evaluate the changes in risk factors over time by subgroups of interest, generalised estimation equations will be used. To estimate the rate of accumulated cardiovascular incidents, the Kaplan-Meier method will be used. The log rank test will be used to compare the differences between the curves of accumulated incidence events. In order to quantify the relationship between risk factors and the incidence of CVD events, the Cox proportional hazards method will be used. Potential confounders and interactions will be explored. Appropriate diagnostics will be carried out to test goodness of fit, collinearity and atypical observations in each model. In all cases, fulfilment of assumptions in the model by means of exploration of residual behaviour will be verified.

Statistical analysis software STATA 10.0 and SAS 9.0 will be used. ${ }^{37-39}$

\section{Ethical aspects}

The study will be carried out following the guidelines for the protection of the rights of human volunteers. All investigators and personnel in the study have completed a training course, certified by the National Institutes of Health (NIH). All participants will sign an informed consent form during the initial visit. To protect participant confidentiality, the information included in the database will not contain personal identifiers.
Timeline of the study

Baseline data collection is projected to be carried out during 2011. The follow-up phase will begin in 2012 and will consist of an annual telephone interview continuing up to the fourth year with a second round of physical and biochemical measurements and an ECG performed 2-3 years after the baseline measurements.

\section{CONCLUSIONS}

Despite the increasing burden of CVD in the Southern Cone, which over the last decades has been ranked as the main cause of mortality and morbidity, national health programs and policies are still mostly focused on interventions aimed at tackling communicable diseases or perinatal or childhood conditions. Therefore, new programs should be targeted at modifying lifestyle and nutritional risk factors to reduce CVD in high risk subjects. ${ }^{36}$ However, the lack of follow-up studies prevents our countries not only from assessing local risk estimates and obtaining more reliable data on the burden of CVD, but also from implementing risk factor stratification and management strategies at a population level. Accordingly, and based on limited and imprecise evidence, there is a strong need in the region to build the capacity and infrastructure necessary to undertake a population-based cohort study to address these remaining knowledge gaps and to inform public health policymakers of the impact of CVD in our countries. The CESCAS I study data will help develop public health strategies based on the application of primary care interventions, thus helping to improve cardiovascular health in this region.

Author affiliations:

${ }^{1}$ Southern Cone American Center for Cardiovascular Health (CESCAS/SACECH), Institute for Clinical Effectiveness and Health Policy (IECS), Buenos Aires, Argentina

${ }^{2}$ Department of Epidemiology, School of Public Health and Tropical Medicine, Tulane University, New Orleans, Louisiana, USA

${ }^{3}$ Sanatorio San Carlos, Cardiología, Bariloche, Rio Negro, Argentina

${ }^{4}$ Universidad de la Frontera, CIGES, Temuco, Chile

${ }^{5}$ Department of Cardiology, Faculty of Medicine, Universidad de la República, Montevideo, Uruguay

${ }^{6}$ Municipalidad de Marco Paz, Secretaría de Salud, Marcos Paz, Argentina ${ }^{7}$ Department of Family Medicine, Faculty of Medicine, Universidad de la República, Montevideo, Uruguay

Acknowledgements We thank Eiman Jahangir and Alisson Lee, fellows of the Fogarty International Clinical Research Fellows Program (FICRF), for careful revision of the manuscript.

Funding This work was supported by the National Heart, Lung, and Blood Institute (NHLBI) grant number HHSN268200900029C.

\section{Competing interests None.}

Ethics approval Institutional review boards from Argentina (Hospital Italiano de Buenos Aires), Chile (Universidad de la Frontera), Uruguay (Universidad de la República) and the USA approved this study.

Contributors AR, JH, VI, RP, LB, FL, JM, MC, HO, PS and JP all contributed to the conceptualisation and design of the study and all revised this manuscript critically. All authors gave final approval of the version to be published.

Provenance and peer review Not commissioned; internally peer reviewed.

Data sharing statement Following the policy of the National Institutes of Health $(\mathrm{NIH})$, we reaffirm the practice of making data used for scholarly research available to other investigators. 


\section{REFERENCES}

1. Gersh BJ, Sliwa K, Mayosi BM, et al. Novel therapeutic concepts: the epidemic of cardiovascular disease in the developing world: global implications. Eur Heart $J$ 2010;31:642-8.

2. Gaziano TA, Bitton A, Anand S, et al. Growing epidemic of coronary heart disease in low- and middle-income countries. Curr Probl Cardiol 2010;35:72-115.

3. Epping-Jordan JE, Galea G, Tukuitonga C, et al. Preventing chronic diseases: taking stepwise action. Lancet 2005;366:1667-71.

4. Abegunde DO, Mathers CD, Adam T, et al. The burden and costs of chronic diseases in low-income and middle-income countries. Lancet 2007;370:1929-38.

5. Chow CK, Lock K, Teo K, et al. Environmental and societal influences acting on cardiovascular risk factors and disease at a population level: a review. Int J Epidemiol 2009;38:1580-94.

6. Yusuf S, Hawken S, Ounpuu S, et al. Effect of potentially modifiable risk factors associated with myocardial infarction in 52 countries (the INTERHEART study): case-control study. Lancet 2004;364:937-52.

7. Murray Cjl LA. The Global Burden of Disease: a comprehensive assessment of mortality and disability from diseases, injuries, and risk factors in 1990 and projected to 2020. Harvard University Press 1996 , World Health Organization The World Health Report: Reducing risks, Promoting Healthy Life. Geneva: World Health Organization, 2002.

8. Lopez AD, Mathers CD, Ezzati M, et al. Global and regional burden of disease and risk factors, 2001: systematic analysis of population health data. Lancet 2006;367:1747-57.

9. Lanas F, Avezum A, Bautista LE, et al. Risk factors for acute myocardial infarction in Latin America: the INTERHEART Latin American study. Circulation 2007;115:1067-74.

10. Rubinstein A, Colantonio L, Bardach A, et al. Estimate of the cardiovascular disease burden attributable to modifiable risk factors in Argentina. Rev Panam Salud Publica ;27:237-45.

11. ENSCH. Encuesta Nacional de Salud en Chile. 2008. http://epi. minsal.cl/epi/html/invest/ENS/ENS_mayo2004.pdf.

12. ENFR. Primera Encuesta Nacional de Factores de Riesgo. Ministerio de Salud de la Nación Argentina, 2006. http://www.msal.gov.ar/htm/ Site/enfr/resultados_completos.asp.

13. ENFRU. Primera Encuesta de Factores de Riesgo de Uruguay, 2006. http://www.sportsalut.com.ar/eventos/cong2007/postcongreso/ jueves13/6GonzaloRodriguez-PrimeraencuestaFRECNTM\%5B1\% 5D\%5B1\%5D.S.pdf.

14. ELSA. ELSA Brasil: the greatest epidemiological study in Latin America. Rev Saude Publica 2009;43.

15. Silva Ayçaguer L. Diseño Razonado De Muestras Y Captación De Datos Para La Investigación Sanitaria. 1st edn. Editorial Díaz de Santos, 2000. Madrid: Díaz de Santos, 2000, p 332.

16. Levy P, Lemeshow S. Sampling of Populations: Methods and Applications. Wiley Series in Survey Methodology, 3rd edn. 1999

17. SOL/HCHS. SOL study of latinos. http://www.cscc.unc.edu/hchs.

18. IPAQ. International Physical Activity Questionnaire. http://www.ipaq ki.se/downloads.htm.

19. GATS. Global Adult Tobacco Survey. http://www.tobaccofreecenter.org.

20. Kroenke K, Spitzer RL, Williams JB. The PHQ-9: validity of a brief depression severity measure. J Gen Intern Med 2001;16:606-13.

21. Bonicatto $S$. Screening of mental disorders in primary care: linguistic adaptation procedure of a diagnostic instrument. Acta psiquiátr psicol Am Lat 1999;45:223-34.
22. Augustovski FA, Irazola VE, Velazquez AP, et al. Argentine valuation of the EQ-5D health states. Value Health 2009;12:587-96.

23. Augustovski FA, Lewin G, Elorrio EG, et al. The Argentine-Spanish SF-36 Health Survey was successfully validated for local outcome research. J Clin Epidemiol 2008;61:1279-84.

24. Perloff D, Grim C, Flack J, et al. Human blood pressure determination by sphygmomanometry. Circulation 1993;88:2460-70.

25. Chobanian AV, Bakris GL, Black HR, et al. The seventh report of the joint national committee on prevention, detection, evaluation, and treatment of high blood pressure: the JNC 7 report. JAMA 2003;289:2560-72.

26. Schargrodsky $\mathrm{H}$, Hernandez-Hernandez R, Champagne BM, et al. CARMELA: assessment of cardiovascular risk in seven Latin American cities. Am J Med 2008;121:58-65.

27. MESA. Multi-Ethnic Study of Atherosclerosis. http://www.mesa-nhlbi. org/.

28. CARDIA. Coronary Aartery Risk Development in Young Adults. http:// www.cardia.dopm.uab.edu/.

29. NHANES. National Health and Nutrition Examination Survey. http:// www.cdc.gov/nchs/nhanes.htm.

30. National Cholesterol Education Program (NCEP) Expert Panel on Detection, Evaluation, and Treatment of High Blood Cholesterol in Adults (Adult Treatment Panel III). Third Report of the National Cholesterol Education Program (NCEP) expert panel on detection, evaluation, and treatment of high blood cholesterol in adults (Adult Treatment Panel III) final report. Circulation 2002;106:3143-421.

31. Expert Committee on the Diagnosis and Classification of Diabetes Mellitus. Report of the expert committee on the diagnosis and classification of diabetes mellitus. Diabet Care 2003;26(Suppl 1): S5-20.

32. Gu D, Wu X, Reynolds K, et al. Cigarette smoking and exposure to environmental tobacco smoke in China: the international collaborative study of cardiovascular disease in Asia. Am J Public Health 2004;94:1972-6.

33. Openclinica. https://community.openclinica.com/.

34. Whelton PK. Epidemiology of hypertension. Lancet 1994;344: 101-6.

35. MacMahon S, Peto R, Cutler J, et al. Blood pressure, stroke, and coronary heart disease. Part 1, Prolonged differences in blood pressure: prospective observational studies corrected for the regression dilution bias. Lancet 1990;335:765-74.

36. Rubinstein A, Colantonio L, Bardach A, et al. Estimation of the burden of cardiovascular disease attributable to modifiable risk factors and cost-effectiveness analysis of preventative interventions to reduce this burden in Argentina. BMC Public Health 2010;10:627.

37. Hosmer D, Lemeshow S. Applied logistic regression. New York, NY: John Wiley \& Sons, Inc, Wiley Series in probability and statistics. 2nd Edition. 2000.

38. Kleinbaum D, Kupper L, Nizam A, et al. Applied Regression Analysis and Multivariable Methods. Duxbury Press, 1998. 3rd edn. 2007.

39. Hosmer D, May S. Applied Survival Analysis: Regression Modeling of Time to Event Data. New York, NY: Wiley Series in Probability and Statistics. 2nd edn. 2008. 